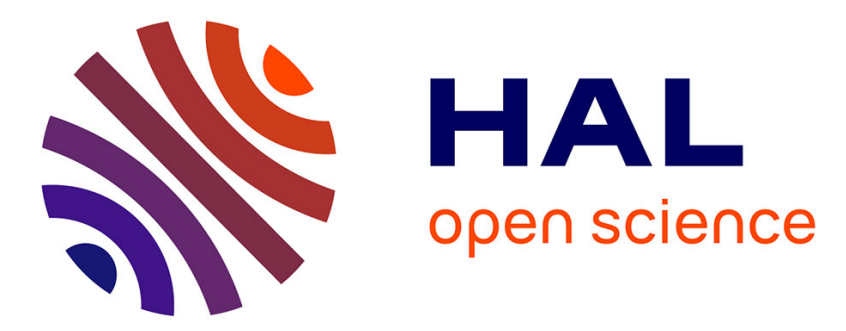

\title{
Les élèves ruraux face à la stigmatisation des territoires
}

Yves Alpe, Angela Barthes

\section{To cite this version:}

Yves Alpe, Angela Barthes. Les élèves ruraux face à la stigmatisation des territoires. Agora débats/jeunesses, 2015. hal-02472004

\section{HAL Id: hal-02472004 https://hal-amu.archives-ouvertes.fr/hal-02472004}

Submitted on 9 Feb 2020

HAL is a multi-disciplinary open access archive for the deposit and dissemination of scientific research documents, whether they are published or not. The documents may come from teaching and research institutions in France or abroad, or from public or private research centers.
L'archive ouverte pluridisciplinaire HAL, est destinée au dépôt et à la diffusion de documents scientifiques de niveau recherche, publiés ou non, émanant des établissements d'enseignement et de recherche français ou étrangers, des laboratoires publics ou privés. 


\title{
Les élèves ruraux face à la stigmatisation des territoires
}

\author{
Yves Alpe, Angela Barthes
}

\section{Introduction}

L'école, en tant que représentation du mythe éducatif républicain, est prise dans une tension permanente entre deux tendances : celle qui, au nom de l'universalisme, voudrait en faire un lieu en dehors du monde où seule triomphe la raison et où ne s'exprime que le mérite, et celle qui la conçoit comme un acteur local, sensible à tous les éléments du contexte économique, social et culturel pour le bien-être de la collectivité dans laquelle elle s'insère. Dans ces conditions, l'école rurale constitue, depuis le $\mathrm{XIX}^{\mathrm{e}}$ siècle, à la fois un idéal l'éducation pour tous, dans chaque village - et un repoussoir, puisqu'elle est souvent accusée de limiter les horizons culturels, de tolérer, sinon d'encourager, le repliement, et de participer ainsi à l'inégalité des chances scolaires.

Même si le débat a changé de tonalité, sous l'effet des transformations socio-économiques et grâce à l'apport des nombreuses recherches qui y ont été consacrées, l'école rurale reste dans les pays développés l'objet d'une stigmatisation quasi institutionnalisée par les politiques éducatives territorialisées (Alpe, 2012 ; Alpe, Barthes, 2010).

Après avoir rappelé le cadre général de ces politiques publiques et la façon dont elles conçoivent le rapport école/territoire, nous montrerons, à partir de l'analyse des résultats scolaires et des trajectoires des élèves ruraux, que les rapports au territoire de ces élèves même s'ils ont beaucoup évolué depuis vingt ans - expriment en partie les effets de cette stigmatisation, ce qui renforce les inégalités d'éducation que ces politiques étaient censées combattre...

\section{École et territoire : des relations ambiguës}

\section{L'école républicaine contre les particularismes locaux}

L'école publique s'est construite contre les territoires. En effet, la volonté de créer une école pour tous s'est traduite par celle d'installer la même école partout, parce qu'elle seule 
pouvait porter les valeurs de la République et le sentiment national. Comme le souligne Antoine Prost (1992, p. 63) : «L'une des fonctions de l'école primaire était de concourir à l'unification des esprits. » Dès lors, les particularismes (dont les «patois ») devaient être éradiqués, et le cadre de référence de tous les élèves devait être le seul cadre national, pour l'étude de la langue comme pour celle de l'histoire (la «civilisation française » dans les anciens manuels) ou de la géographie (qui enseigne les «frontières naturelles » du territoire...). Par la mise en place de la forme scolaire, ont été réalisées les conditions d'un déracinement qui devait faciliter l'intégration à la communauté nationale : «Depuis la Révolution, le modèle français se réclame d'un corps politique unifié et aménage le territoire de façon centralisatrice, affirmant la primauté de la capitale et des pouvoirs qui y résident. L'appartenance primordiale sinon unique à la "nation" est inculquée à l'école » (Bérard et al., 2005, p. 11.). Par là même, elle est censée offrir à tous les mêmes chances, par le biais d'une conception méritocratique de l'école. Les écoles rurales, plus tardivement implantées et plus tardivement fréquentées, incarnent alors parfaitement ce combat et ces enjeux, puisque ce sont évidemment les ruraux qui sont le plus éloignés des valeurs que veut porter cette école de la modernité (Alpe, Fauguet, 2008).

La réalisation de ce programme n'est pas allée sans résistances, et les enseignants l'ont très souvent détournée ou limitée.

De cette façon, le territoire a pu être reconnu comme une opportunité pédagogique et didactique, facilitant les apprentissages et développant la motivation des élèves. De nombreux mouvements pédagogiques (à l'image de l'école Freinet) se sont réclamés de cette posture, qui s'accompagne généralement d'une grande attention portée aux relations locales (avec les élus locaux, le mouvement associatif...). On voit bien que, comme dans le cas précédent, c'est l'acquisition de la culture scolaire qui est au centre des préoccupations toute la question étant de savoir laquelle. Et la façon de trancher dans ce débat est assez évidente, dans son principe, sinon dans sa méthode : il faut savoir ce qui fait réussir les élèves.

\section{La géographie de l'école et l'inégalité des chances scolaires}

Pour l'école rurale, la problématique va changer dans les années 1960 sous les effets conjugués de l'exode rural (avec les difficultés du maintien des services publics en zone rurale) et de l'émergence d'une vision critique de l'école, portée par la sociologie de l'éducation (Bourdieu, Passeron, 1970 ; Baudelot, Establet, 1975), qui met l'accent sur les inégalités en matière de réussite scolaire. Dès lors, la thématique récurrente devient celle des 
«handicaps socioculturels »-c'est vrai pour l'école rurale comme pour les familles populaires, et ces dernières sont très nombreuses en milieu rural... -, et l'institution scolaire cherche à savoir si les élèves ruraux sont «défavorisés ». Beaucoup de recherches sur ce sujet vont voir le jour dans les années 1980 et 1990, et elles vont nourrir (de façon souvent très détournée) le positionnement des politiques publiques d'éducation qui se centrent sur trois objectifs :

- Regrouper : le « handicap » est supposé d'autant plus grand que l'école est petite et isolée ; il faut donc supprimer les plus petites ${ }^{1}$. Cette politique peut s'appliquer aussi au collège rural et permet d'économiser des postes d'enseignants...

- Compenser : il faut apporter aux élèves ce qui leur manque du fait de leur isolement scolaire, social et culturel. On met en place de nombreux systèmes de compensation, très souvent appuyés sur les technologies de communication et le multimédia qui font entrer la modernité dans la moindre école rurale...

- Adapter : cet objectif, défendu par la Délégation interministérielle à l'aménagement du territoire et à l'attractivité régionale (DATAR) a rencontré peu d'écho dans l'Éducation nationale, qui campe sur ses positions en faveur d'une école unique pour tous. Néanmoins, des expériences intéressantes ont vu le jour, en particulier dans l'enseignement professionnel (comme des formations à la pluriactivité en montagne).

L'institution scolaire a tenté à plusieurs reprises d'évaluer les résultats de ces politiques, par exemple à travers les rapports de l'inspection générale de l'Éducation nationale (IGEN, voir encadré 1). Mais ces tentatives ont eu très peu d'effet, soit parce qu'elles ne faisaient que reprendre les a priori sur le «déficit culturel » (rapport Lebossé), soit parce que les observations des ces rapports sont restées sans suite lorsqu'elles n'allaient pas dans le sens des choix politiques en la matière (rapport Duhamel). Ce sont donc principalement les recherches sur les performances scolaires des élèves ruraux qui ont nourri le débat.

\section{Encadré 1. Les principaux rapports de l'inspection générale de l'Éducation nationale}

- Le rapport Mauger (Agir ensemble pour l'école rurale, 1992) vante les mérites de l'école à trois classes et propose de supprimer les autres par regroupements ; il fixe une taille minimale souhaitable pour les collèges.

- Le rapport Ferrier (consacré aux « réseaux scolaires en milieu rural », 1995) reprend la même

\footnotetext{
${ }^{1}$ De 1960 à 2000, le nombre d'écoles a diminué de 34,7\% et le nombre d'écoles à classe unique de 70,5\% (source : Ministère de l'Éducation nationale, Direction de l'évaluation et de la prospective - MEN/DEP).
} 
idée et préconise, comme son nom l'indique, la mise en réseau des écoles.

- Le rapport Lebossé (Pour une nouvelle dynamique du système éducatif en zone rurale isolée, 1998) reprend une fois encore la même thématique autour de la proposition de création des « réseaux d'écoles rurales » qui fera l'objet d'un texte officiel en 1999.

- Le rapport Duhamel (L'évolution du réseau des écoles primaires, 2003), qui constitue de facto la suite du précédent, fait le bilan de la mise en place des réseaux, avec des remarques sur l'ouverture de l'école, déjà largement réalisée, ou sur l'insuffisance de l'analyse des conséquences pédagogiques des différentes mesures - par exemple, sur les regroupements : "Aucune démonstration sérieuse ni étude valide ne permet de démontrer que la réussite scolaire est meilleure »; sur les réseaux : «Il faut oser dire que le choix d'organisation en réseau n'a, au mieux, qu'une incidence indirecte sur les résultats scolaires. » Pourtant, le rapport recommande en conclusion « un service minimum obligatoire garanti d'au moins trois classes couvrant les trois cycles » (ce que conseillait déjà le rapport Mauger).

Il est à noter que le rapport Duhamel est le dernier de la série, si l'on excepte un rapport plus spécialisé (Le plan École numérique rurale (Rapport $\mathrm{n}^{\circ}$ 2011-073, juin 2011, IGENIGAENR) et qui reprend en partie la problématique habituelle : «situation difficile des écoles rurales» (p. 11), faiblesse des moyens des petites communes, etc. Il apporte cependant une précision très intéressante: «Le plan ENR [École numérique rurale] a entraîné une sorte de "double fracture numérique", d'abord, au sein du milieu rural, entre les écoles dotées et celles qui ne l'ont pas été, faute soit d'être éligibles, soit d'être candidates ; ensuite, entre les petites écoles rurales bien équipées et les agglomérations plus importantes, chefs-lieux de cantons ou de départements, qui ne disposent que de postes informatiques traditionnels, parfois obsolètes, placés dans une salle dédiée ou au fond de la classe » (p. 20). Cette constatation confirme, presque mot pour mot, une des conclusions des enquêtes de l'OER, qui datent, pour la première, de 1999.

\section{Les élèves ruraux sont-ils défavorisés ?}

\section{Quelques données nationales sur les performances scolaires des ruraux}

Les travaux de l'Institut national des études démographiques (Girard, Bastide, Pourcher, 1970) sur les élèves de CM2 en 1962 constituent la première mesure des inégalités géographiques en matière de réussite scolaire. Ils montrent des écarts énormes dans les taux d'accès à la classe de sixième (40\% des garçons à la campagne, $72 \%$ à Paris). Les enquêtes suivantes (nombreuses dans les années 1980-1990) sont plus optimistes pour le primaire : à l'école, les différences entre élèves urbains et ruraux concernant les apprentissages 
fondamentaux sont faibles, et, en milieu rural, ce sont les écoles à trois classes qui obtiennent les meilleurs résultats. Mais les inégalités demeurent : le taux d'accès en seconde (Euvrard, 2003) varie avec la taille des collèges ruraux (40,3\% pour ceux de moins de 200 élèves, 52,6 \% pour ceux de plus de 400 élèves) et l'insertion dans le second cycle général long est plus difficile pour les élèves ruraux, qui s'orientent plus que proportionnellement à leurs résultats vers l'enseignement professionnel.

Ces résultats seront affinés par l'évaluation globale du système éducatif en milieu rural, publiée par la Direction de l'évaluation et de la prospective (DEP) en 1995 (CEuvrard, 1995). L'examen comparatif des résultats bruts des tests d'évaluation au CE2 indique que les élèves des écoles rurales obtiennent des scores légèrement plus élevés que ceux des écoles urbaines, même si les écarts globaux sont trop faibles pour être jugés significatifs. En revanche, lorsque l'on passe des résultats observés aux résultats attendus, c'est-à-dire lorsque l'on intègre les caractéristiques sociodémographiques des familles concernées, ils obtiennent un score global de 1,4 point (sur 100), supérieur à celui des écoles urbaines (1,6 point en mathématiques et 1,2 point en français).

De plus, les résultats des élèves ayant fréquenté des classes à cours multiples (classes à quatre ou cinq niveaux) sont nettement meilleurs que la moyenne, surtout en mathématiques (CEuvrard, 1995).. Françoise Euvrard (2003) signale aussi que, tant en CE2 qu'en sixième, les résultats sont plus homogènes : la différence de réussite entre enfants d'ouvriers et de cadres est plus faible chez les ruraux que chez les urbains.

Par contre, l'analyse des orientations en fin de troisième conduit à des conclusions moins optimistes : les élèves des collèges ruraux demandent moins que leurs homologues urbains une classe de seconde et davantage l'enseignement professionnel court (CAP, BEP, apprentissage) et se jugent plus sévèrement: les écarts entre leur choix initial et leur orientation effective sont moindres que chez les élèves urbains.

\section{Les enquêtes de l'OER ${ }^{2}(1999-2006)$}

L'Observatoire de l'école rurale (OER) a mené de 1999 à 2006 une série d'enquêtes par suivi de cohorte sur 2400 élèves ruraux de six départements français, en questionnant aussi leurs parents. Une nouvelle enquête (2011-2012) portant sur 1200 élèves de CM2 (2011) et

\footnotetext{
${ }^{2}$ L'OER a élargi son champ d'investigations et s'appelle aujourd'hui OET (Observatoire éducation et territoires). Il est rattaché scientifiquement à l'EA 4671 ADEF (Apprentissage, didactique, évaluation, formation) de l'université d'Aix-Marseille.
} 
959 élèves de troisième (2012) est en cours d'exploitation.

Globalement, ces enquêtes montrent peu de changements notoires dans l'origine sociale (il y a une très légère remontée des catégories favorisées), mais les familles du rural restent plus modestes que la moyenne nationale: en 1999, $20 \%$ des pères dans la base OER appartenaient aux catégories «favorisées » ou «assez favorisées », contre $32,1 \%{ }^{3}$ pour l'ensemble de la France.

Les travaux de l'OER (Alpe, Champollion, Poirey, 2001-2010) confirment cependant que les élèves ruraux réussissent bien à l'école. Ils sont un peu moins souvent en retard à l'entrée en sixième, et enregistrent de meilleurs scores aux tests d'évaluation.

Tableau 1. Résultats des tests d'évaluation en sixième (2000)

\begin{tabular}{|l|l|c|c|}
\hline & & Français & Mathématiques \\
\hline \multirow{3}{*}{ Score $>90$} & OER & $8 \%$ & $10 \%$ \\
\cline { 2 - 4 } & National & $5,8 \%$ & $8,1 \%$ \\
\hline \multirow{2}{*}{ Score $<30$} & OER & $1 \%$ & $3 \%$ \\
\cline { 2 - 4 } & National & $2,4 \%$ & $5,9 \%$ \\
\hline
\end{tabular}

Source : base OER (2 600 élèves ruraux) et résultats nationaux ${ }^{4}$.

La comparaison entre la base OER et les résultats nationaux donne un léger avantage aux élèves de la base OER, dans les deux domaines et dans les deux sens - les «bons »sont un peu plus nombreux et les «faibles» un peu moins -, ce qui ne fait que confirmer les résultats globaux des études de la DEP déjà évoquées. Enfin, à l'issue de la première année au collège, $6 \%$ des élèves suivis par l'OER redoublent, ce qui est conforme aux chiffres nationaux et aux taux des milieux urbains.

Et pourtant, les élèves ruraux ont tendance à minorer leurs capacités et performances lorsqu'on les interroge sur leur niveau scolaire. Dans la façon dont ils se jugent, ils sont plus pessimistes que leurs homologues urbains, alors que leurs résultats objectifs sont plutôt légèrement meilleurs.

Tableau 2 : opinion des élèves de troisième sur leur propre niveau scolaire (\%)

\begin{tabular}{|l|c|c|}
\hline & Urbains & Ruraux \\
\hline Excellent & 5 & 3 \\
\hline Bon & 38 & 31 \\
\hline
\end{tabular}

\footnotetext{
${ }^{3}$ Données du Panel CP/97 du MEN : voir Note d'information, nº 98-40, MEN/DPD, décembre 1998.

${ }^{4}$ Note d'information, ${ }^{\circ}$ 01-36, MEN/DPD, juillet 2001.
} 


\begin{tabular}{|l|c|c|}
\hline Moyen & 38 & 45 \\
\hline Rencontrant des difficultés & 19 & 21 \\
\hline Total & $\mathbf{1 0 0}$ & $\mathbf{1 0 0}$ \\
\hline
\end{tabular}

Source : enquêtes OER 2004.

Cette «modestie relative » se retrouve dans les recherches menées par Françoise Euvrard, dans les enquêtes approfondies sur les élèves ruraux du département de l'Ain en 1993 (Poirey, Fromajoux, 1998), et on peut aussi la relever dans les enquêtes récentes (2011 et 2012) de l'OET sur 1200 élèves ruraux des Alpes de Haute-Provence, de l'Ardèche et de la Drôme. Mais comment l'expliquer?

\section{Les rapports au territoire des élèves ruraux}

\section{Les opinions sur le territoire}

Dans les enquêtes menées par l'OET, les élèves étaient amenés à exprimer leur choix pour leur futur milieu de vie. Lorsqu'on observe la «balance des opinions » (les réponses positives moins les réponses négatives), les résultats sont sans ambiguïté : les opinions positives vis-à-vis de la «campagne » se réduisent fortement dans le temps. Cela se vérifie lorsque les mêmes élèves sont interrogés à deux périodes différentes (ici CM2 et troisième) et dans les enquêtes successives auprès des élèves de CM2 (ici 1999 et 2011) et de troisième (2004 et 2012).

\footnotetext{
Graphique 1. «Où je souhaiterais exercer mon futur métier », balance des opinions, CM2 1999 et troisième 2004
} 


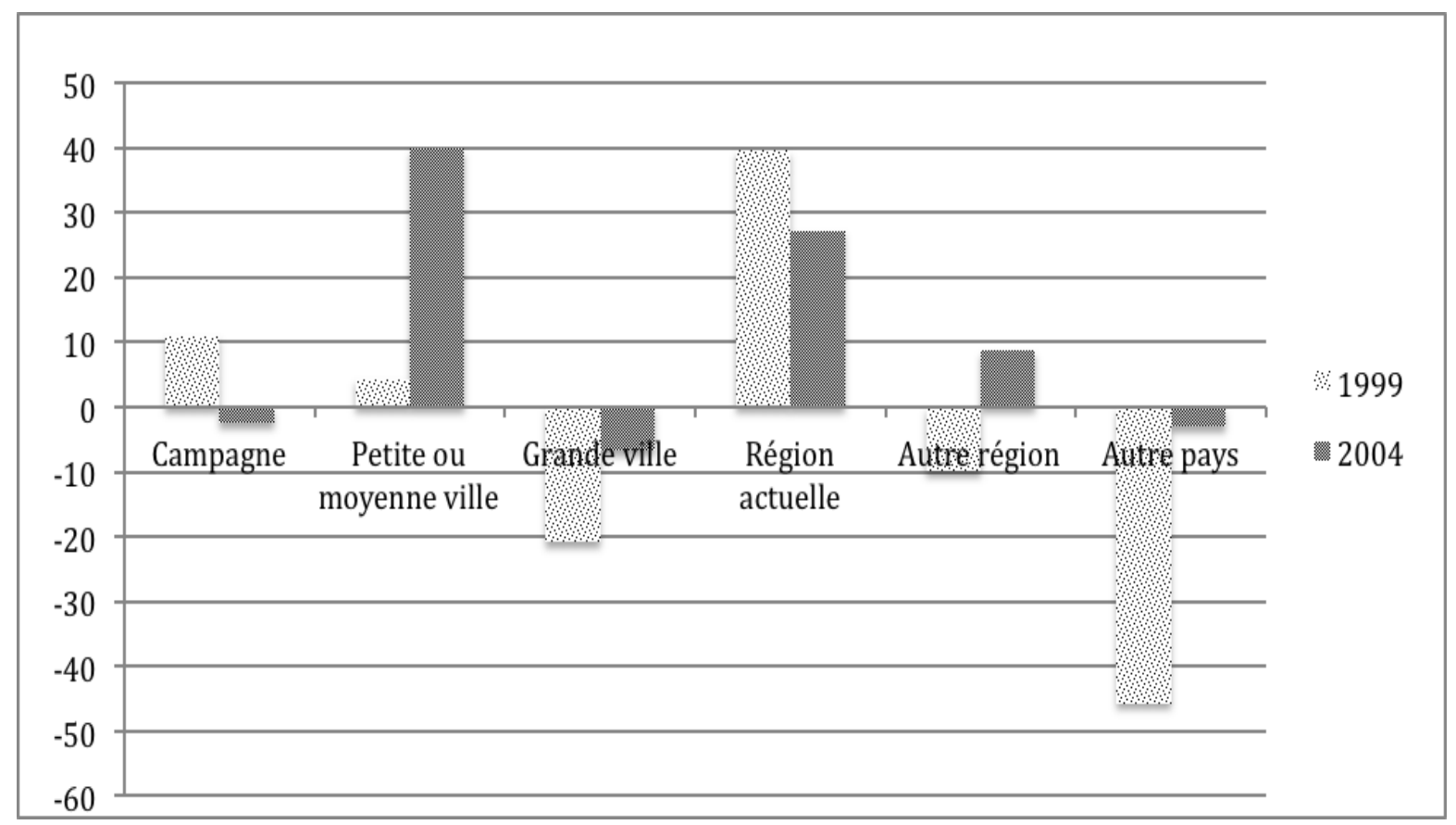

Source : enquêtes OER-OET, 1364 élèves ruraux interrogés en CM2, puis en troisième.

Lecture : la balance des opinions concernant la campagne (\% d'opinions positives - \% d'opinions négatives) est positive en 1999, ce n'est plus le cas en 2004.

Les réponses montrent clairement le recul des opinions favorables relatives à la campagne et la diminution de la répulsion pour la mobilité géographique.

Graphique 2. « Où je souhaiterais exercer mon futur métier », balance des opinions, CM2 1999 (2 634 élèves) et CM2 2011 (1 208 élèves)

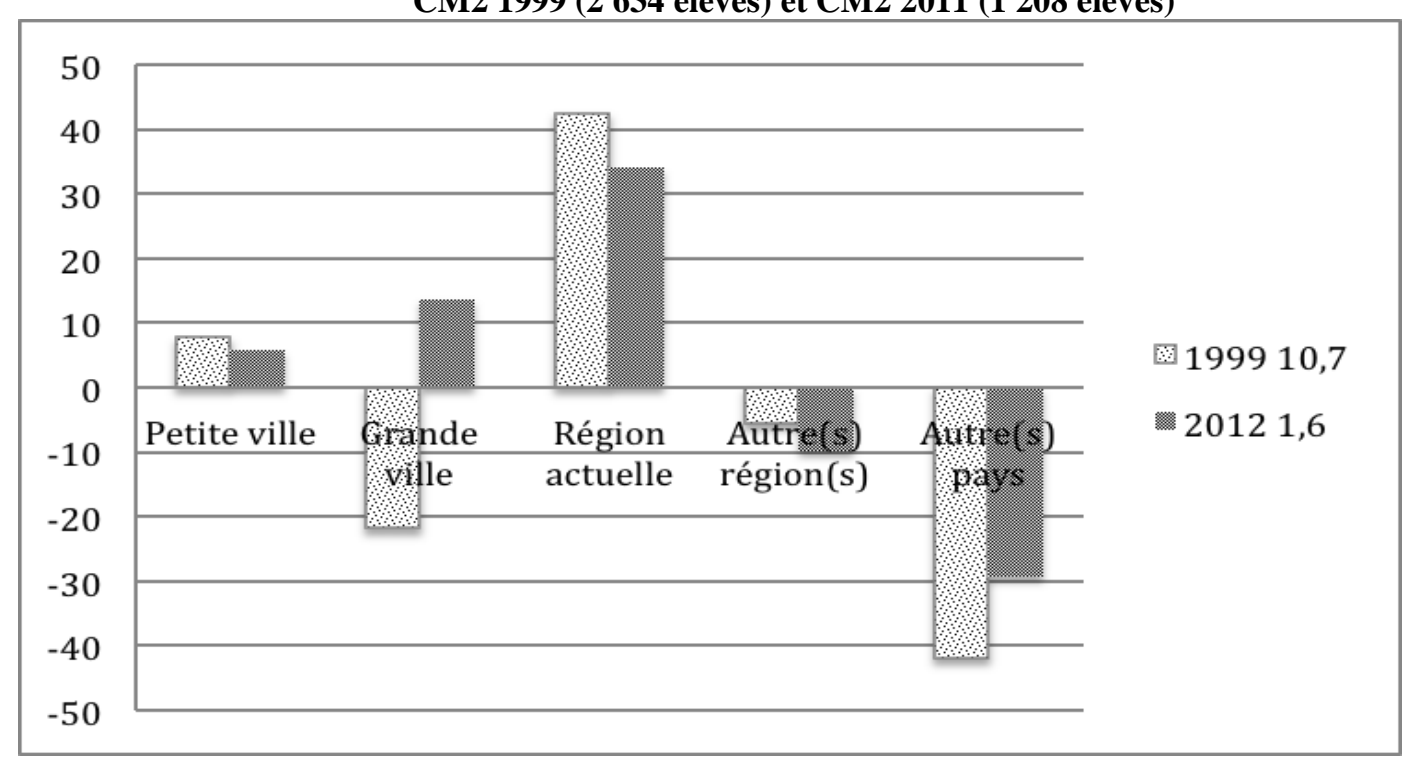

Enquêtes OER-OET 1999 et 2011.

Lecture : les opinions positives pour la campagne l'emportent, mais plus faiblement en 2011 : la différence entre le $\%$ d'opinions positives et le $\%$ d'opinions négatives est de $+10 \%$ en 1999, elle est inférieure à $2 \%$ en 2011. 
La comparaison dans le temps donne des résultats similaires : la campagne est de moins en moins attractive, et la grande ville, répulsive en 1999, est devenue attractive en 2011. Et tous les éléments vont dans le même sens. Les métiers ruraux attirent de moins en moins les élèves, le collège rural est jugé à l'aune d'une «modernité » attribuée au collège urbain... En bref, les élèves des écoles rurales présentaient en 1999 un a priori favorable pour la campagne, qui a disparu aujourd'hui, alors que la montée des préoccupations environnementales aurait pu faire penser le contraire... Les élèves ont donc intégré à leur discours non seulement une autodépréciation (ils se jugent «moins bons » que les élèves urbains, à résultats égaux ou même supérieurs), mais aussi majoritairement un rejet de leur milieu de vie pour leur propre avenir.

L'évolution est encore plus marquée pour les élèves de troisième, comme on peut le voir sur le graphique 3.

Graphique 3. « Où je souhaiterais exercer mon futur métier », balance des opinions, troisième 2004 (1 364 élèves) et troisième 2012 ( 954 élèves)

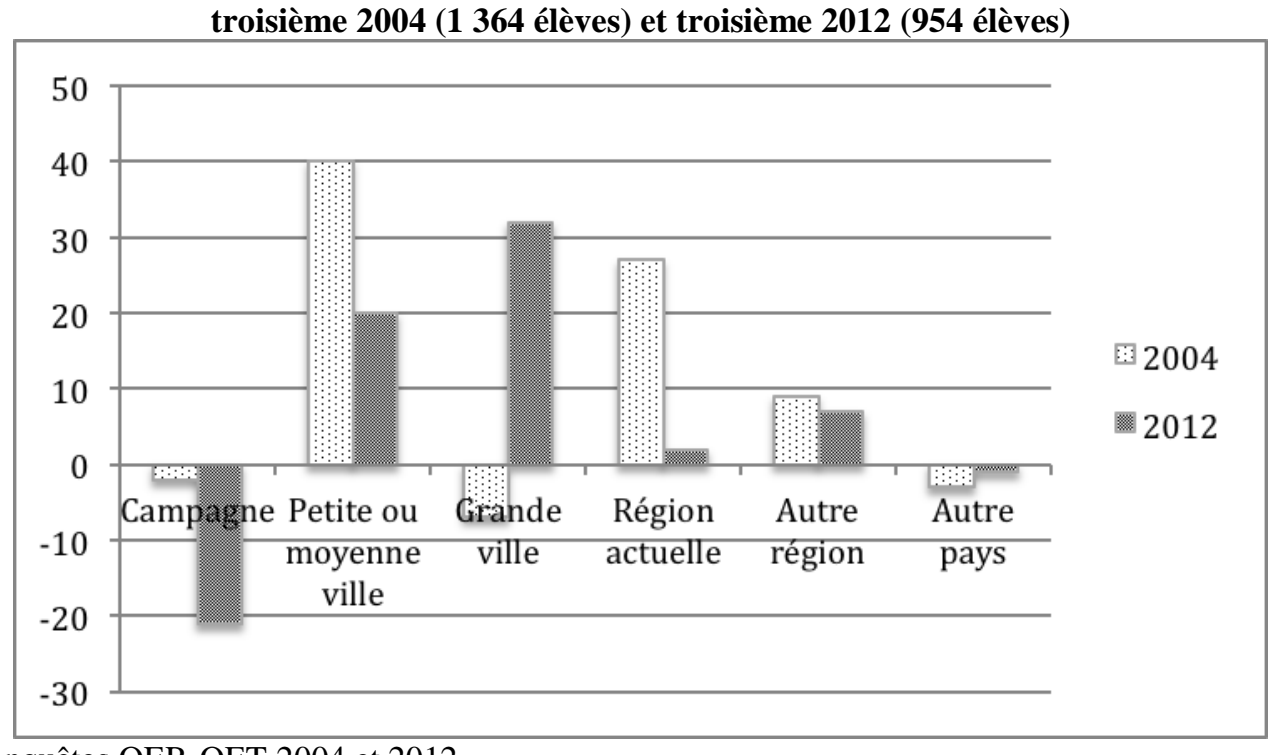

Source : enquêtes OER-OET 2004 et 2012.

Lecture : la balance des opinions (\% d'opinions positives - \% d'opinions négatives) est fortement négative $(-21 \%)$ en 2012 : la plupart des élèves de troisième refusent d'exercer leur futur métier à la campagne.

Par ailleurs, l'analyse des réponses de ceux qui envisagent d'aller travailler dans un «autre 
pays » (dernière colonne du graphique 3) fait apparaître des choix typiques des adolescents (États-Unis en tête, et aucun effet de la proximité géographique). Ce sont donc des choix « rêvés », il n’y a pas d'incidence du « rural » dans leurs projets.

Et si l'on analyse les réponses ouvertes des élèves, on voit que les deux principaux éléments de la dépréciation relative de la «campagne » sont d'ordre social et culturel : petite taille des réseaux sociaux, faiblesse des équipements socioculturels, manque d'opportunités diverses en matière de loisirs par exemple.

\section{Les projets scolaires et professionnels des élèves de troisième}

Les élèves de troisième ont déjà une idée assez précise de leur avenir scolaire et professionnel, toutes les études le confirment. Les enquêtes de l'OER entre 1999 et 2004 avaient montré une réduction progressive de leurs ambitions au cours de leur scolarité, le pourcentage de ceux qui envisageaient des études supérieures chutant de moitié entre l'entrée en sixième et l'entrée en seconde, pour les élèves « à l'heure ».

Les résultats de 2012 ne confirment pas ce déficit relatif d'ambition: alors que les bacheliers 2011 se sont inscrits pour 74,6 \% à l'université ${ }^{5}, 80,2 \%$ des élèves de troisième interrogés avaient l'intention de s'y inscrire. Certes, les données ne sont pas comparables (inscriptions contre intentions), mais les ruraux montrent une fois de plus qu'ils sont dans la tendance nationale, avec toutefois une préférence plus marquée $(39,1 \%$ contre $34 \%)$ pour l'enseignement court.

Nous disposons là encore de deux enquêtes (2004 et 2012) auprès de collégiens ruraux (autrement dit fréquentant des collèges classés en zone rurale). Les élèves étaient amenés à exprimer deux projets, un « réaliste » et un «rêvé ».

Tableau 3. Métiers souhaités (« rêvés ») en \% du total des réponses

$\begin{array}{lclc} & \mathbf{2 0 0 4} & & \mathbf{2 0 1 2} \\ \text { Chanteur } & 13,6 & \text { Scientifique, chercheur } & 6,8 \\ \text { Acteur } & 13,4 & \text { Ingénieur } & 6,6 \\ \text { Footballeur } & 9,9 & \text { Puériculteur } & 6,6 \\ \text { Médecin } & 8,2 & \text { Médecin } & 5,9 \\ \text { Pilote d'avion } & 7,1 & \text { Cuisinier } & 5,6 \\ \text { Avocat } & 6,5 & \text { Vendeur } & 5,6 \\ \text { Astronaute } & 6,4 & \text { Architecte } & 5,4 \\ \text { Ingénieur } & 5,7 & \text { Vétérinaire } & 4,9 \\ \text { Professeur } & 4,7 & \text { Professeur } & 4,7 \\ \text { Nombre de réponses } & 1288 & & 425\end{array}$

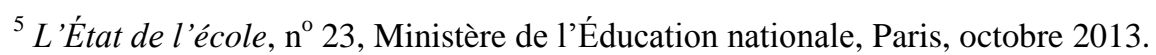


Source : élèves de troisième (collèges ruraux), Enquêtes OER-OET 2004 et 2012 (en grisé les métiers présents dans les deux listes).

Tableau 4. Métiers « réalistes » en \% du total des réponses

$\begin{array}{lclc} & \mathbf{2 0 0 4} & & \mathbf{2 0 1 2} \\ \text { Professeur } & 19,5 & \text { Vendeur } & 7,3 \\ \text { Vendeur } & 6,4 & \text { Ingénieur } & 7,1 \\ \text { Puériculteur } & 6,0 & \text { Architecte } & 6,3 \\ \text { Boulanger pâtissier } & 6,0 & \text { Cuisinier } & 5,9 \\ \text { Coiffeur } & 5,8 & \text { Puériculteur } & 5,9 \\ \text { Infirmier } & 5,2 & \text { Coiffeur } & 4,9 \\ \text { Informaticien } & 5,0 & \text { Scientifique, chercheur } & 4,9 \\ \text { Ingénieur } & 4,3 & \text { Boulanger pâtissier } & 4,6 \\ \text { Esthéticien } & 4,2 & \text { Médecin } & 4,6 \\ \text { Éducateur } & 3,9 & \text { Mécanicien } & 4,1 \\ \text { Nombre de réponses } & 1494 & & 222 \\ \text { \% du total des réponses } & 66,3 & & 55,6\end{array}$

Source : élèves de troisième (collèges ruraux), enquêtes OER-OET 2004 et 2012 (en grisé les métiers présents dans les deux listes).

Ces deux tableaux présentent les dix premières occurrences de chaque liste. Là encore, la comparaison doit s'effectuer avec prudence, car le nombre de réponses est beaucoup plus faible (l'échantillon est plus petit) en 2012, et la dispersion des réponses plus grande. Mais, il y a, cependant, des faits notables.

Tout d'abord, la «ruralité » ne transparaît nullement (sauf peut-être avec le métier de vétérinaire) dans ces réponses, qui sont à peu de chose près celles des adolescents français de cette tranche d'âge, ce que confirmait déjà la comparaison entre collèges ruraux et urbains effectuée par l'OER en 2004.

Mais le plus frappant est la comparaison dans le temps. Alors que les élèves de 2004 distinguaient très nettement les métiers «rêvés » et «réalistes », ceux de 2012 ne rêvent plus ! Leurs choix sont très semblables et témoignent (d'autres éléments de l'enquête le confirment) de préoccupations très pragmatiques et modestes: dans les dix premières occurrences, les artisans et employés représentent 6 réponses sur 10, alors que ces élèves ont connu des scolarités sans problème pour la plupart.

En clair, les élèves ruraux ont une appréciation assez réaliste des possibilités qui s'ouvrent à eux : le choix d'orientation vers l'enseignement professionnel correspond aux opportunités offertes par leur territoire (Arrighi, 2004), et la nécessité de migrer est de plus en plus 
acceptée. Il faut d'ailleurs souligner que, contrairement aux idées reçues, les élèves ruraux sont «mobiles ». En 1999, 46,8 \% des élèves de CM2 de la base OER n'étaient pas nés dans leur département de scolarisation, et les jeunes ruraux interrogés en 2011 au CM2 (1 208 élèves, base OET) avaient déjà déménagé une fois au moins pour $84 \%$ d'entre eux, et $33,5 \%$ plus de deux fois.

\section{Les politiques éducatives territorialisées : un diagnostic erroné, des remèdes qui aggravent le mal}

\section{Le rural vu par l'institution scolaire}

On l'a évoqué ci-dessus, l'institution scolaire a une vision très construite du rural, qu'elle reprend et répète sans cesse, comme le montrent les rapports de l'inspection générale de l'Éducation nationale (IGEN). De 1992 (rapport Mauger) à 2003 (rapport Duhamel), presque rien n'a changé dans le discours. Le diagnostic que posaient ces rapports est fondé sur une série d'affirmations, que l'on peut illustrer par quelques extraits du rapport Lebossé (encadré 2), car la position de l'institution, non révisée sur le fond depuis 2003, n'a que très peu évolué depuis.

\section{Encadré 2 - Le rapport Lebossé}

(Pour une nouvelle dynamique du système éducatif en zone rurale isolée, 1998)

«Les activités périscolaires, culturelles et sportives sont peu développées, voire inexistantes, par manque de moyens des collectivités locales et parce que de telles activités sont difficiles à mettre en œuvre pour des effectifs très réduits. [...] Les élèves issus des collèges ruraux se dirigent plus fréquemment vers des études courtes et professionnelles et fréquentent donc moins les lycées d'enseignement général ou technologiques, mais la dimension socioculturelle du rural en est sans doute l'explication majeure. [...] Dans le rural isolé, la continuité entre les étapes successives du système éducatif est moins bien appréhendée par les familles en raison d'un éclatement géographique des différents établissements et d'une demande sociale d'éducation moins forte. [...] Mais, du fait de l'isolement de leurs habitants, les zones concernées peuvent ne pas voir émerger de projets. »

Le problème, c'est que ce rapport, qui a largement inspiré les suivants, ne fournit absolument aucune preuve de ce qu'il avance. Les «activités périscolaires », contrairement à ce qui est écrit, sont très présentes en milieu rural, mais elles le sont souvent par l'intermédiaire d'acteurs locaux ignorés de l'institution scolaire. La «dimension socioculturelle du rural » n'est en aucune façon explicitée ou analysée, et renvoie en fait à la 
vieille idée du « handicap socioculturel » des ruraux, que les politiques dites «d'ouverture » avaient pour but de combattre. Or, les enquêtes de l'OER ont clairement montré que ces politiques n'avaient aucune incidence sur la réussite scolaire ou les trajectoires des élèves ruraux. Enfin, l'affirmation d'une demande d'éducation moins forte est contraire à tous les résultats de recherche, et témoigne d'une certaine ignorance des réalités de terrain : il suffit d'observer la virulence des conflits locaux en matière d'éducation (fermetures de classes, absences d'enseignants ou d'options...) pour s'en convaincre. Quant à «l'isolement des habitants », il renvoie à un monde d'avant les technologies de communication et la « reruralisation ». L'exemple d'autres contextes, comme celui du Québec (Pévost, 2004), montre que l'isolement peut être aussi une motivation pour monter des projets.

\section{Inégalités scolaires et stigmatisation des territoires}

Aujourd'hui, force est de constater que les politiques éducatives territorialisées ont manqué leur objectif. D’abord, parce que celui-ci était mal défini : au motif de réduire les inégalités des chances scolaires entre les ruraux et les urbains, on a mis en place des dispositifs censés rapprocher l'école rurale de l'école urbaine. Il faut redire, comme le reconnaisssait le rapport Duhamel, qu'ils n'ont eu aucun effet positif - si ce n'est sur le plan purement financier : ils ont réduit les coûts unitaires de l'école rurale -, et ils ont alourdi les charges pesant sur les collectivités locales (les transports scolaires en particulier).

Dans le même temps, le recentrage sur les «fondamentaux» (le français et les mathématiques) et le durcissement des règles administratives ont détruit l'un des bénéfices les plus précieux de l'école rurale : celle-ci remplissait à la fin des années 1990 un rôle très important de compensation des inégalités en matière de pratiques culturelles (l'accès au théâtre par exemple), ce qui n'est plus le cas aujourd'hui. Un seul élément a sans doute joué en faveur des écoles rurales : le développement de l'éducation à l'environnement et au développement durable (Bader, Sauvé, 2011), encouragé par de nombreux textes, a pu être vu comme une opportunité pour les écoles rurales en prise directe avec leur environnement et souvent entourées d'acteurs locaux qui y sont très attachés. La revalorisation de l'environnement peut, dans une certaine mesure, lutter contre la stigmatisation des espaces ruraux si l'on arrive à éviter les écueils des représentations naïves de la « nature ».

De plus, bien évidemment, la stigmatisation des territoires n'est pas sans produire des effets pervers. En encourageant le départ des jeunes, on dévitalise d'importantes portions du territoire, on multiplie les contraintes (déplacements) qui vont à l'encontre des préoccupations environnementales affichées par ailleurs... 
Enfin, le «mauvais territoire »- le rural, la zone d'éducation prioritaire (ZEP) - sert aussi à dissimuler d'autres rapports de force et d'autres inégalités : dans le rural comme ailleurs, et peut-être même plus qu'ailleurs, le principal facteur de l'inégalité des chances scolaires reste l'origine socioculturelle (profession et catégorie socioprofessionnelle [PCS] du père et diplôme de la mère). En reportant dans le discours la responsabilité des écarts constatés sur le territoire, on se dispense de s'attaquer à ce qui constitue, aujourd'hui comme hier, le défaut essentiel du système éducatif français, son incapacité, encore rappelée par les dernières enquêtes PISA, à réduire l'effet de l'origine sociale sur la réussite scolaire.

\section{Conclusion}

Les jeunes ruraux ont aujourd'hui, pour l'essentiel, les mêmes références culturelles que leurs homologues urbains, et beaucoup de prises de position semblent ignorer que pour bon nombre de nos contemporains les territoires sont devenus «virtuels»: ce sont ceux des technologies de communication et des réseaux sociaux, qui s'affranchissent des contraintes géographiques (le temps des réseaux filaires, si difficiles à développer à la campagne, est derrière nous). Ils rencontrent aussi les mêmes difficultés : l'insertion socio-économique suppose non seulement un diplôme, mais aussi des opportunités d'emploi. Si elles sont faibles, l'école n'y peut rien.

Mais, de ce point de vue, ils ont un avantage. Il ne fait aucun doute que la lecture de l'espace social de l'action est beaucoup plus facile dans une petite communauté où les liens sont forts. Et cela permet de mettre en œuvre, quand on connait assez finement ce qu'offre le territoire, des stratégies complexes. Ainsi, les élèves de collèges ou de lycées ruraux (il en existe !) interrogés par l'OER ont souvent exprimé un choix de «partir-revenir ». Partir pour les études, pour voir le monde, et revenir parce qu'il existe un attachement au milieu dans lequel on a vécu - même si ce n'est plus aujourd'hui, dans la majorité des cas, un milieu d'origine-, et parce que les réseaux locaux facilitent l'insertion pour l'emploi, le logement...

Enfin, il ne faut pas oublier que l'école rurale a constitué un laboratoire de l'innovation pédagogique, parce qu'il fallait prendre en compte des contraintes moins connues ailleurs (classes multi-niveaux par exemple). Des savoir-faire existent, ils sont souvent trop peu valorisés, ou même complètement ignorés : les modules de formation consacrés à l'école rurale ou à la classe unique, fréquents dans les instituts universitaires de formation des maîtres (IUFM) dans les années 1990, ont connu une spectaculaire régression ensuite, alors même que l'on tentait d'importer en ville des modèles expérimentés dans le rural (classes 
multi-niveaux en ZEP). Il faut espérer que la nouvelle formation des maîtres qui se met en place saura tirer parti de ces atouts.

\section{Références bibliographiques}

Alpe Y., «Contexte territorial et organisation scolaire : l'école rurale française, de l'idéalisation à la stigmatisation », Revue suisse des sciences de l'éducation, $\mathrm{n}^{\circ} 34,2012 / 2$, pp. 213-232.

Alpe Y., Barthes A., «Les aspects socio-géographiques de l'évolution de l'éducation publique en milieu rural et montagnard», in Alpe Y ., Champollion P., Poirey J.-L., L'enseignement scolaire en milieu rural et montagnard. Tome V. Après le collège, Presses universitaires de Franche-Comté, Besançon, 2010, pp. 149-170.

Alpe Y., Champollion P., Poirey, J.-L. (coord.), L'enseignement scolaire dans les milieux ruraux et montagnards. Tomes I, II, III, IV, V, Presses universitaires de Franche-Comté, Besançon, 2001, 2003, 2005, 2006, 2010.

Alpe Y., Fauguet J.-L., Sociologie de l'école rurale, L’Harmattan, coll. «Éducations et sociétés », Paris, 2008.

Arrighi J.-J., «Les jeunes dans l'espace rural : une entrée précoce sur le marché du travail ou une migration probable », Formation Emploi, n 87,2004 , pp. 63-78.

Bader B., Sauvé L., Éducation, environnement et développement durable : vers une écocitoyennté critique, Presses de l'Université Laval, coll. «L'espace public », Québec (Canada), 2011.

Baudelot C., Establet R., L'école primaire divise, Maspéro, coll. «Petite collection Maspéro », Paris, 1975.

Bérard L. et al., Les notes de l'IDDRI, «Savoirs et savoirs faire naturalistes locaux : l'originalité française $», n^{\circ} 7,2005$.

Bourdieu P., Passeron J.-C., La reproduction. Éléments pour une théorie du système d'enseignement, Minuit, coll. «Le sens commun », Paris, 1970.

Girard A., Bastide H., Pourcher G., « Enquête nationale sur l'entrée en sixième et la démocratisation de l'enseignement », in Population » et enseignement, 1970, Presses universitaires de France, Paris, pp. 51-90.

Euvrard, F. (coord), Éducation et formations, «Le système éducatif en milieu rural », $\mathrm{n}^{\circ} 43$, octobre 1995, pp. 5-155.

Euvrard, F., «Les "performances" de l'école "rurale". Quelle mesure, dans quel objectif ? », Villeécole-intégration Enjeux, «Le rural : terre d'exclusion? », $\mathrm{n}^{\circ}$ 134, septembre 2003, pp. 151-164.

Prévost P., Projet L'École éloignée en réseau. Les collaborations école-communauté au Québec: une perspective de développement local au moyen de quatre études de cas, Rapport de recherche, 
version finale 2004, CEFRIO, Québec (Canada), 2004.

Poirey J.-L., Fromajoux R.-C., L'école rurale, au carrefour des territoires et des réseaux, Presses universitaires de Lyon, coll. « Travaux et documents », Lyon, 1998.

Prost A., Éducation, société et politiques. Une histoire des enseignements en France de 1945 à nos jours, Le Seuil, Paris, 1992.

Prost A., Histoire générale de l'enseignement et de l'éducation en France. Tome IV.e L'École et la famille dans un société en mutation (depuis 1930), Perrin, Paris, 2004.

\section{Résumé :}

L'école rurale est l'objet depuis plus d'un siècle de débats sur sa capacité à proposer aux élèves une scolarité conforme aux attentes du modèle républicain de l'école publique. Les recherches sur les trajectoires scolaires des élèves ruraux ont montré la bonne tenue de leurs résultats, elles ont aussi souligné la modestie relative de leurs ambitions. Face à l'hétérogénéité croissante du «rural» et à la généralisation des modèles culturels, les politiques publiques, axées sur la réduction d'un prétendu «déficit socioculturel », se sont avérées inefficaces, et parfois même pénalisantes, dans la mesure où elles participent à la stigmatisation des territoires ruraux.

\section{Les auteur·e·s}

Yves Alpe

yves.alpe@univ-amu.fr

Professeur émérite, EA 4671 ADEF, université d'Aix-Marseille, Observatoire éducation et territoires.

Thèmes de recherche: sociologie de l'éducation, inégalités territoriales d'éducation, épistémologie des savoirs scolaires

A notamment publié

Alpe, Y., Fauguet J.-L., Sociologie de l'école rurale, L'Harmattan, coll. « Éducation et Sociétés », Paris 2008.

Alpe, Y., « Le curriculum sournois du développement durable », in Bader B., Sauvé L. (dir.), Éducation, environnement et développement durable : vers une écocitoyenneté critique, Presses de l’Université Laval., Québec (Canada), 2011, pp.103-122.

Alpe Y., «Contexte territorial et organisation scolaire : l'école rurale entre idéalisation et stigmatisation », Revue suisse des sciences de l'éducation, $\mathrm{n}^{\circ} 2$, vol. XXXIV, 2012, pp. 213231. 
Maître de conférences HDR, IUT d'Aix-Marseille EA 4671 ADEF, université d'AixMarseille, ses thèmes de recherche portent sur les contextes et enjeux éducatifs du développement. Elle s'est particulièrement intéressée aux «éducations à » (au développement durable, au patrimoine, aux territoires), et aux spécificités éducatives des espaces ruraux.

Elle a notamment publié :

A. BARTHES et P. CHAMPOLLION, (dir), (2014), L'école rurale et montagnarde en contexte méditerranéen : Approches socio-spatiales. Volume 6. Besançon. Presses universitaires de Franche-Comté. 220 p.

A. BARTHES, Y. ALPE, (2014), Le curriculum caché du développement durable, Revue Penser l'éducation, Philosophie de l'éducation et histoires des idées pédagogiques, horssérie, http://shs-app.univ-rouen.fr/civiic/index.php?id=51

B. BADER, A. BARTHES, A. LEGARDEZ, (2013), Rapport aux savoirs, éducation relative à l'environnement et au développement durable, Education Relative à l'Environnement: Regards - Recherches - Réflexions" volume 11, Québec http://www.revue-ere.uqam.ca

A. BARTHES, P. CHAMPOLLION, (2012), Éducation au développement durable et territoires, Evolution des problématiques, modification des logiques éducatives, et spécificité des contextes ruraux. Education relative à l'environnement, Regards. Recherches. Réflexions, $\quad \mathrm{n}^{\circ} 10, \quad$ Québec, $\quad$ pp.36-51. 6-51 http://www.revueere.uqam.ca/categories/volumes/v10.html

A. BARTHES, A. JEZIORSKI, A. LEGARDEZ, (2012), What kind of critical university education for sustainable development? A comparative study of European students and social representations, in the epistemological and didactical challenges involved in teaching socially acute questions, Journal of Social Science of Education: Volume 11, Number 4 http://www.jsse.org/index.php/jsse/article/view/79

A. BARTHES, Y. ALPE, (2012), Les «éducations à », un changement de logique éducative? L'exemple de l'éducation au développement durable à l'université, Spirale, $\mathrm{n}^{\circ}$ 50, Les éducations à... : nouvelles recherches, nouveaux questionnements ? 2012. pp. 197209.http://spirale-edu-revue.fr/spip.php?article1114 